\title{
Trends and Applications for Cognitive Radio
}

\author{
Enrico Del Re, ${ }^{1}$ Torleiv Maseng, ${ }^{2}$ and Luca Simone Ronga ${ }^{3}$ \\ ${ }^{1}$ Department of Electronics and Telecommunications, University of Florence, Via di Santa Marta 3, 50139 Florence, Italy \\ ${ }^{2}$ Forsvarets Forskningsinstitutt, P.O. Box 25, 2027 Kjeller, Norway \\ ${ }^{3}$ Italian National Consortium for Telecommunications (CNIT), Via di Santa Marta 3, 50139 Firenze, Italy \\ Correspondence should be addressed to Enrico Del Re, enrico.delre@cnit.it
}

Received 27 July 2012; Accepted 27 July 2012

Copyright (c) 2012 Enrico Del Re et al. This is an open access article distributed under the Creative Commons Attribution License, which permits unrestricted use, distribution, and reproduction in any medium, provided the original work is properly cited.

After a decade of cognitive radio, it is time to check the level of maturity and the real gains attained by this technology. Born with many expectations, which is the real status of its deployment? From one side a sensible slowing down of the standardization process is perceived, probably due to economical reasons rather than technical issues. On the other side the demand of spectral efficiency for boosting performances lowering energy consumption is rapidly increasing. The feeling is that the latter driver prevails against the former, and this is proven by an increased scientific and industrial interest on efficient CR solutions.

The special issue describes some of the most relevant technological trends and promising applications of the CR idea.

The paper entitled "The SS-SCR scheme for dynamic spectrum access" describes an interesting connection between a sense-based resource allocation and a cooperation scheme for a multihop, multichannel, and multi PU cognitive environment. The scheme can be implemented in a cloudcomputing environment, relevant to a consolidated technological trend for future urban deployments.

The paper entitled "Cognitive code-division channelization with admission control" combines a resource allocation strategy with an admission control procedure, revealing all the inner complexity this process owns. The proposed solution is interesting also for its energy efficiency, proving that a satisfactory compromise between efficiency and performance is one of the achievements of CR.

The paper entitled "Optimizing cooperative cognitive radio networks with opportunistic access" deeply explores the adoption of cooperation for opportunistic radio access. Several configurations of relays and direct accesses are studied and compared, giving a complete view of the capability of the cooperative paradigm.

The paper entitled "Optimal pricing of spectrum resources in wireless opportunistic access" represents here a wide class of relevant studies for $\mathrm{CR}$, that is, the adoption of Game Theory to model the selfish behavior of secondary nodes only partially aware of the complete spectral environment. The paper provides an in-depth study of the advantages of the taxation of used resources in terms of local and global throughput.

The paper entitled "Hybrid experiential-heuristic cognitive radio engine architecture and implementation" implements the resources decision process by integrating the two main approaches, an heuristic one with a learning-based one. The resulting scheme is studied and experimented on a real HW platform to prove its capabilities in terms of flexibility and spectral efficiency.

The paper entitled "On spectrum sensing for TV white space in china" enters the details of a possible exploitation of TV white spaces. The paper reports an experimental HW implementation of the spectrum sensing module for the Chinese context, revealing the global applicability of TVWS concept.

The paper entitled "Cognitive scout node for communication in disaster scenarios" presents an interesting application of CR in emergency scenarios. The proposal is based on a scout node with two main functions: the establishment of a detailed radio spectrum map in the theatre of operations and a cooperative base-station aiding the primary network during emergency.

The selected papers show recent theoretical advances toward CR adoption, but also real experimental setups that prove the high degree of maturity of this technology for mass 
deployment. The negative energy balance due to increased complexity in the $\mathrm{CR}$ receivers is compensated by the improved spectral efficiency attained by recent CR schemes.

Enrico Del Re

Torleiv Maseng

Luca Simone Ronga 

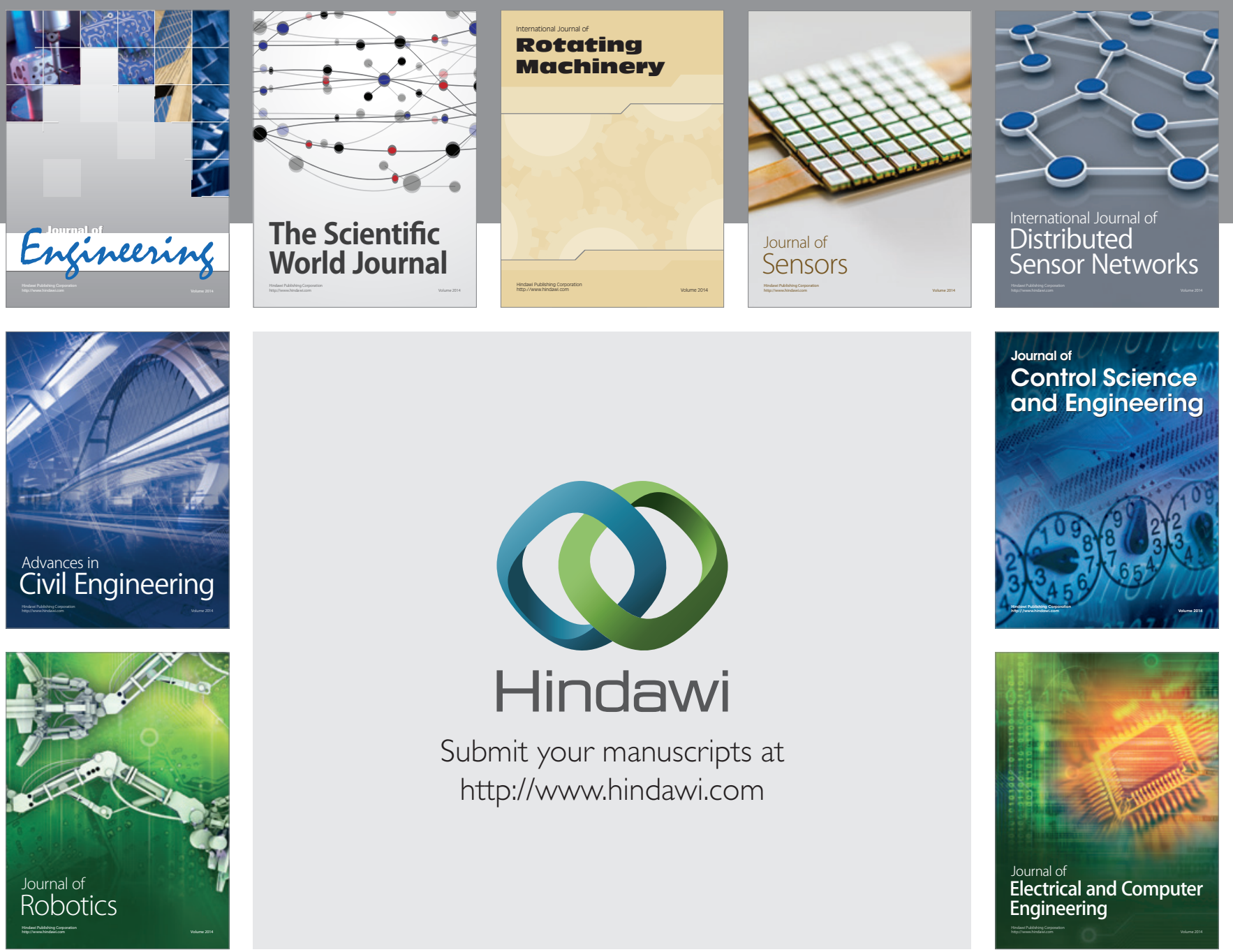

Submit your manuscripts at

http://www.hindawi.com
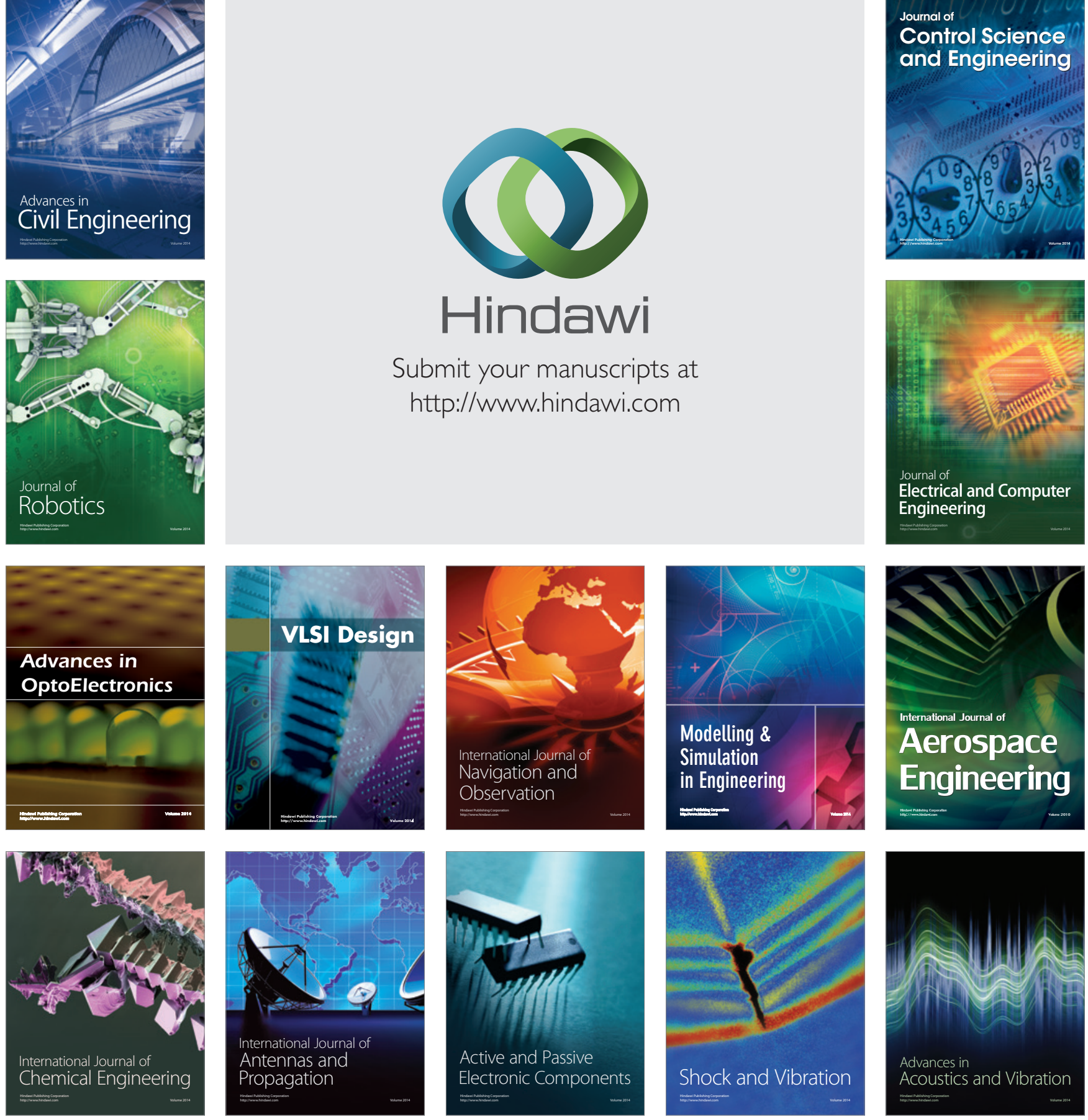